\title{
CD147/Basigin Deficiency Prevents the Development of Podocyte Injury through FAK Signaling
}

Tomoki Yoshioka, ${ }^{* \dagger}$ Tomoki Kosugi, ${ }^{*}$ Tomohiro Masuda, ${ }^{*}$ Tomoharu Watanabe, ${ }^{* \dagger}$ Akihiro Ryuge, ${ }^{* \dagger}$ Hiroshi Nagaya, ${ }^{*}$ Kayaho Maeda, ${ }^{*}$ Yuka Sato, ${ }^{*}$ Takayuki Katsuno, ${ }^{*}$ Noritoshi Kato, ${ }^{\star}$ Takuji Ishimoto, ${ }^{*}$ Yukio Yuzawa, ${ }^{\S}$ Shoichi Maruyama, ${ }^{*}$ and Kenji Kadomatsu ${ }^{\dagger}$

From the Departments of Nephrology* and Biochemistry, ${ }^{\dagger}$ Nagoya University Graduate School of Medicine, Nagoya; the Department of Nephrology and Rheumatology, ${ }^{\ddagger}$ Aichi Medical University, Nagakute; and the Department of Nephrology, ${ }^{\S}$ Fujita Health University School of Medicine, Toyoake, Japan

\author{
Accepted for publication \\ April 2, 2019. \\ Address correspondence to \\ Tomoki Kosugi, M.D., Ph.D. \\ Department of Nephrology, \\ Nagoya University Graduate \\ School of Medicine, 65 \\ Tsurumai-cho, Showa-ku, \\ Nagoya 466-8550, Japan. \\ E-mail: kosugi@med.nagoya-u. \\ ac.jp.
}

\begin{abstract}
Podocytes, which are susceptible to injury by various stimuli and stress, are critical regulators of proteinuric kidney diseases, regardless of the primary disease and pathogenesis. We further confirmed a significant correlation between urinary CD147/basigin (Bsg) levels and proteinuria in patients with focal segmental glomerulosclerosis. However, the molecular mechanism of podocyte injury involving Bsg is not fully understood. Here, the involvement of Bsg in the pathogenesis of podocyte injury was elucidated. Healthy podocytes rarely express Bsg protein. In two independent mouse models, including adriamycin-induced nephropathy and $\mathrm{N}^{\omega}$-nitro-L-arginine methyl ester (L-name)-induced endothelial dysfunction, Bsg induction in injured podocytes caused podocyte effacement, which led to development of proteinuria. Bsg silencing in cultured podocytes exposed to transforming growth factor- $\beta$ suppressed focal adhesion rearrangement and cellular motility via the activation of $\beta_{1}$ integrin-focal adhesion kinase-matrix metallopeptidase signaling. In addition, induction of vascular endothelial growth factor and endothelin-1, which are implicated in podocyte-to-endothelial cross-communication, was lower in the supernatants of cultured Bsg-silenced podocytes stimulated with transforming growth factor- $\beta$. In this setting, Bsg may be involved in a physiological positive feedback loop that accelerates podocyte cell motility and depolarization. The current study thus suggests that Bsg silencing via suppression of $\beta_{1}$ integrin-focal adhesion kinase-matrix metallopeptidase signaling may be an attractive therapeutic strategy for the maintenance of podocytes in patients with proteinuric kidney diseases. (Am J Pathol 2019, 189: 1338-1350; https://doi.org/10.1016/j.ajpath.2019.04.003)
\end{abstract}

Proteinuria, the clinical manifestation of glomerular filtration barrier dysfunction, is a critical determinant of the progression of chronic kidney disease and cardiovascular diseases. ${ }^{1-4}$ Podocytes contribute to the maintenance of an adequate intracapillary environment by molecular signaling crosstalk with glomerular endothelial cells and the extracellular matrix. ${ }^{5,6}$ In particular, precise regulation of vascular endothelial growth factor (VEGF) derived from podocytes is essential for maintenance of endothelial function. The extracellular matrix serves as a storage compartment for signaling molecules that maintain a healthy glomerular balance. Matrix metalloproteinases (MMPs) promote disruption of the glomerular basement membrane (GBM), turnover of extracellular matrix, and disruption of podocyte integrity. ${ }^{7-9}$ When intraglomerular homeostasis is disrupted, the skewed profile of cytokine expression causes loss of the size/charge selectivity of glomerular filtration, leading to development of persistent proteinuria. Therefore, further identification of these regulators, as well as an enhanced understanding of

\footnotetext{
Supported in part by Grant-in-Aid for Progressive Renal Diseases Research, Research on Rare and Intractable Disease, Nephrology Research from the Ministry of Health, Labor and Welfare of Japan grant 90584681 (T.K.).

Disclosures: None declared.
} 
Table 1 Clinical Characteristics of Patients with FSGS

\begin{tabular}{lcr}
\hline Clinical characteristics & FSGS $(N=95)$ & Pathologic control $(N=23)$ \\
\hline Sex, male, \% & 54.7 & 39.1 \\
BMI, kg/m ${ }^{2}$ & $19.8 \pm 5.1$ & $17.6 \pm 5.5$ \\
Systolic BP, mm Hg & $137 \pm 18.3$ & $117 \pm 20.4$ \\
Diastolic BP, mm Hg & $80 \pm 13.0$ & $69 \pm 12.4$ \\
eGFR, mL/min/1.73 m ${ }^{2}$ & $64.2 \pm 22.5$ & $89.0 \pm 24.8$ \\
UP/urine Cr & $3.35 \pm 3.06$ & $0.10 \pm 0.15$ \\
Hb, mg/dL & $13.5 \pm 2.14$ & $13.9 \pm 1.71$ \\
\hline
\end{tabular}

Data are expressed as means \pm SD.

BMI, body mass index; $\mathrm{BP}$, blood pressure; $\mathrm{Cr}$, creatinine; eGFR, estimated glomerular filtration rate; FSGS, focal segmental glomerulosclerosis; Hb, hemoglobin; UP, urine protein.

already established signaling, is required for therapeutic intervention in patients with proteinuric kidney diseases.

Bsg encodes CD147/basigin (Bsg), which is an extracellular MMP inducer and a highly glycosylated, transmembrane, immunoglobulin superfamily member protein. ${ }^{10}$ Bsg is expressed in many cell types, such as hematopoietic, epithelial, and endothelial cells, and is critically important in processes such as invasion by cancer cells, spermatogenesis, viral infection, and immune system regulation. ${ }^{11-15}$ Generation of Bsg-deficient mice $\left(\mathrm{Bsg}^{-/-}\right)$in our laboratory ${ }^{16,17}$ showed that Bsg induces cytokine production and regulates neutrophil and macrophage migration in models of ischemic renal injury and renal fibrosis. ${ }^{18,19}$ In addition to a role in eliciting chemotactic activity, Bsg also serves as a negative immune modulator in IL-17-producing T-cell differentiation in lupus nephritis. ${ }^{20}$ In a series of clinical studies, ${ }^{21-23}$ a striking increase in Bsg in the circulating blood and urine was observed in patients with acute kidney injury, lupus nephritis, and persistent kidney diseases with proteinuria. Regardless of the primary kidney disease or pathogenesis, a significant relationship between proteinuria and urinary CD147/Bsg levels was observed in proteinuric kidney diseases. However, the involvement of Bsg in the molecular mechanism of proteinuric kidney diseases has not yet been elucidated in detail.

In accordance with previous reports, Bsg is involved in the production of VEGF, MMPs, and hyaluronan, and in the interaction with $\beta_{1}$ integrin, all of which increase our understanding of the complex scenario regarding glomerular filtration barrier dysfunction. ${ }^{24-27}$ In this study, we focused on Bsg functions in proteinuric kidney diseases induced by doxorubicin hydrochloride (ADR) or $\mathrm{N}^{\omega}$-nitro-L-arginine methyl ester (L-name). It was then investigated whether Bsg deficiency reduced podocyte cell motility by inhibiting focal adhesion turnover, thereby preventing the development of proteinuria.

\section{Materials and Methods}

\section{Patients and Procedures}

From 2008 to 2014 at Nagoya University and affiliated hospitals, 95 adults who underwent a kidney biopsy as part of standard of care for the differential diagnosis of various renal diseases (52 males; 43 females) were enrolled. These
95 patients received a final diagnosis of focal segmental glomerulosclerosis (FSGS) because they satisfied the criteria of the Kidney Disease Improving Global Outcomes guidelines. Patients diagnosed with cancer, severe infections, and other renal disorders were excluded. Patients underwent biopsy before any medical treatment began. Also, between 2008 and 2014, 23 pathologic control patients (9 males; 14 females) with only microhematuria or minimal proteinuria, which are not classified as kidney diseases according to clinical and pathologic criteria, were enrolled. All procedures were performed as described previously. ${ }^{23}$

This study was conducted as stated by the principles of the Declaration of Helsinki, the Japanese National Ethical Guidelines (approval number 1135), and the institutional review boards of Nagoya University Hospital and affiliated hospitals. Before participating in the study, each patient provided written informed consent.

\section{Animals and Experimental Design}

Mice deficient in Bsg $\left(\mathrm{Bsg}^{--}\right)$were rarely born following ordinary mating, and thus, a protocol was established for producing sufficient numbers of pups. ${ }^{16} \mathrm{Bsg}^{+/-}$mice on the 129/SV background were backcrossed with C57BL/6J mice to produce F1 hybrid offspring (reverse F1 hybrid). By intercrossing these mice, mixed reverse F2 mice were generated and used in this study. For the adriamycin model, 8- to 12 -week-old male mice weighing 25 to $30 \mathrm{~g}$ were administered a single intravenous injection of ADR (Wako Pure Chemical, Osaka, Japan) at a dosage of $16 \mathrm{mg} / \mathrm{kg}$ body weight. ${ }^{28}$ An equal volume of $0.9 \%$ saline was injected into mice as a control. Mice were sacrificed 2 weeks after treatment. For the L-name model, 8- to 12-week-old male mice weighing 20 to $30 \mathrm{~g}$ were given $1 \mathrm{mg} / \mathrm{mL}$ L-name (Sigma-Aldrich, St. Louis, MO) in their drinking water for 12 weeks $^{29}$ and then sacrificed. Blood and urine samples and kidneys were removed for examination. Serum creatinine $(\mathrm{Cr})$, the ratio of albumin to $\mathrm{Cr}$ in urine, and systolic blood pressure were measured as described previously. ${ }^{30,31}$ Plasma and urinary Bsg levels were measured using commercial enzyme-linked immunosorbent assay kits according to the respective instructions from the manufacturers (R\&D Systems, Minneapolis, MN). Measured levels in urine were 

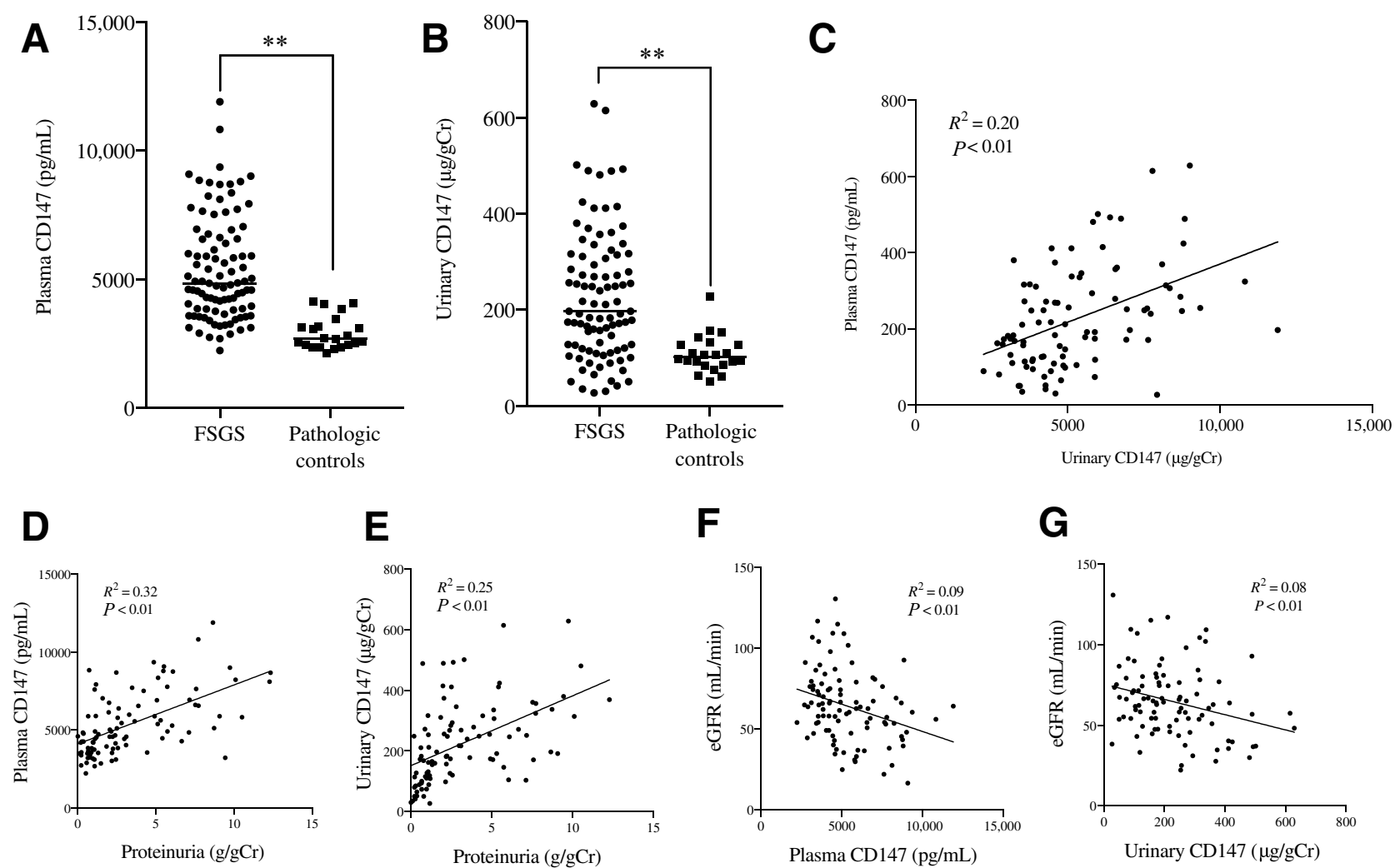

$\mathbf{F}$

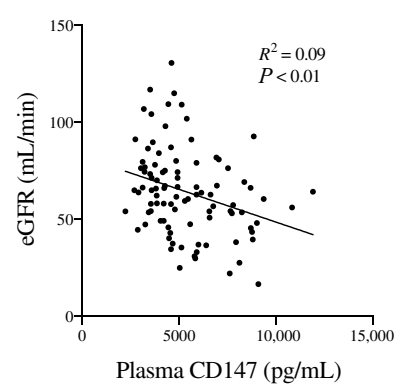

G

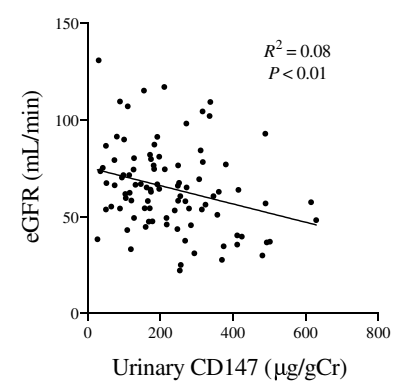

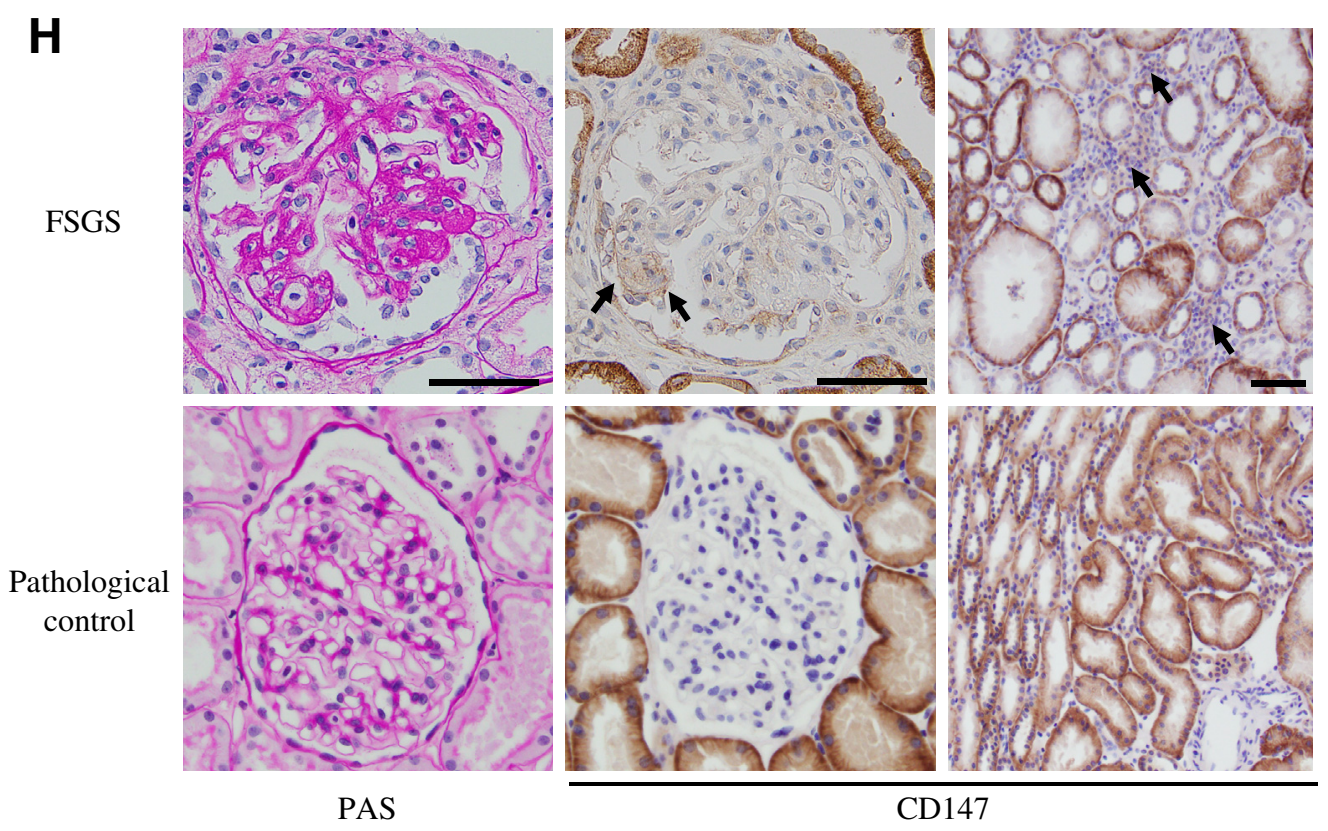

I

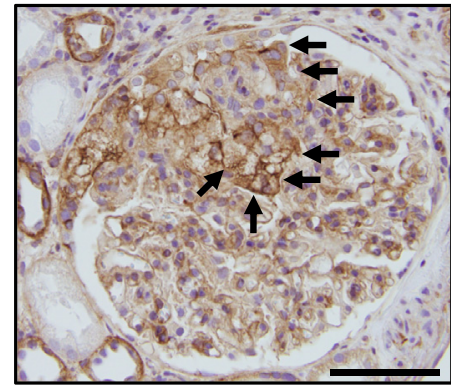

$\beta_{1}$ Integrin

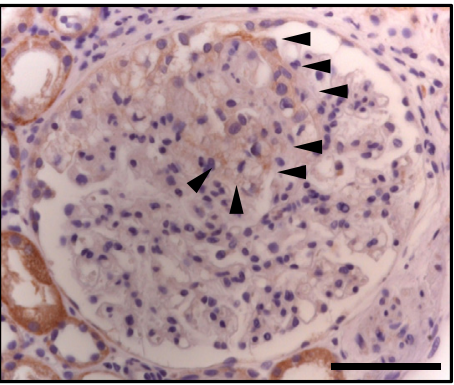

CD147 
then normalized to urinary $\mathrm{Cr}$ levels. All experiments with mice were conducted as specified by the animal experimentation guidelines of Nagoya University School of Medicine.

\section{Renal Histology}

Kidneys were incubated in $10 \%$ formalin, embedded in paraffin, and then sectioned at a thickness of $1 \mu \mathrm{m}$ (periodic acid-Schiff reagent staining) or $4 \mu \mathrm{m}$ (immunohistochemistry). The sections were stained with mouse monoclonal anti-human CD147 antibody (Ab; Abcam, Cambridge, MA), rabbit monoclonal anti-human $\beta_{1}$ integrin $\mathrm{Ab}$ (Abcam), goat antimouse Bsg Ab (R\&D Systems), and rabbit anti-mouse WT-1 Ab (Santa Cruz Biotechnology, Dallas, TX), followed by detection using a peroxidase-conjugated secondary antibody (Nichirei, Tokyo, Japan). The staining was visualized with 3,3'-diaminobenzidine (Dako, Carpinteria, CA), which produces a brown color. Podocytes positive for WT-1 were counted by examining all glomeruli of the cortex under a microscope at high magnification. Quantitation was performed by two experts (T.M. and K.M.) in nephropathology who were blinded to the experimental conditions.

For transmission electron microscopic analysis, kidneys were fixed in formalin, embedded in epoxy resin, sectioned, and then stained with uranyl acetate and lead citrate. As described by Koop et al, ${ }^{32}$ the total length of the GBM and the number of slit pores were evaluated in the free capillary wall, and then the average foot process width was examined by calculating the ratio of the total length of the GBM to the total number of slits.

\section{In Situ Hybridization}

The mice were perfused and fixed with G-Fix (GenoStaff Co., Tokyo, Japan), and kidneys were dissected, embedded in paraffin with G-Nox (GenoStaff Co.), and then sectioned at 8 $\mu \mathrm{m}$. Paraffin-embedded sections were hybridized with sense (https://www.ncbi.nlm.nih.gov/nuccore; accession number NM_009768.2) and antisense Bsg probes. In situ hybridization was performed with the ISH Reagent kit according to the manufacturer's instructions (GenoStaff Co.). The sections were incubated with anti-digoxygenin alkaline phosphatase conjugate (Roche Diagnostics, Mannheim, Germany), and color reactions were developed with NBT/ BCIP solution (Sigma-Aldrich). For immunostaining as the second stain, the sections were incubated with rabbit anti- mouse WT-1 Ab and incubated with biotin-conjugated goat anti-rabbit IgG (Dako), followed by addition of peroxidaseconjugated streptavidin (Nichirei). Peroxidase activity was visualized with $3,3^{\prime}$-diaminobenzidine.

\section{Real-Time PCR}

Mouse glomeruli were dissected from frozen renal sections using the LMD CM 1950 system (Leica Biosystems, Nussloch, Germany). ${ }^{33}$ The RNeasy Micro Kit (Qiagen, Hilden, Germany) was used to isolate total RNA from these samples. Real-time PCR was conducted using the Applied Biosystems Prism 7500HT sequence detection system and TaqMan gene expression assays (Applied Biosystems, Foster City, CA). The TaqMan probes and primers were: Bsg (Mm01144228_g1), endothelin (ET)-1 (Edn1: Mm0043656_m1), transforming growth factor (TGF)- $\beta$ (Tgfbl: Mm01178820_m1), and glyceraldehyde 3-phosphate dehydrogenase (Gapdh: Mm99999915_g1). Applied Biosystems Sequence Detection software version 1.3.1 was used for analysis.

\section{Western Blot Analysis}

Mouse renal tissues and podocytes grown in vitro were lyzed in radioimmunoprecipitation assay buffer (Santa Cruz Biotechnology). Western blot analysis was conducted as described. ${ }^{34}$ After separation of proteins and transfer to membranes, the membranes were incubated with goat anti-mouse nephrin $\mathrm{Ab}$ (R\&D Systems), goat anti-human Bsg Ab, mouse anti-human focal adhesion kinase (FAK) Ab (BD Biosciences, San Diego, CA), and mouse anti-human cellular fibronectin (FN) $\mathrm{Ab}$ (Abcam). Membranes were washed and incubated with peroxidase-conjugated anti-goat $\mathrm{IgG}$, mouse IgG, or rabbit IgG (Jackson ImmunoResearch Laboratories, West Grove, PA). An enhanced chemiluminescence detection system (GE Healthcare, Buckinghamshire, UK) was used to visualize Ab-bound proteins. The density of each band was quantitated using ImageJ software version 1.48 (NIH, Bethesda, MD; https:// imagej.nih.gov/ij).

\section{Cell Culture}

Conditionally immortalized human podocytes obtained from Prof. Richard J. Johnson at the University of Colorado were maintained as described previously. ${ }^{35}$ Cells were incubated at $37^{\circ} \mathrm{C}$ for 7 days to induce differentiation and then transfected using lipofectamine (Thermo Fisher

Figure 1 CD147/basigin expression in patients with focal segmental glomerulosclerosis (FSGS). Scatter plots display the data for plasma (A) and urinary CD147/basigin (B) values in individual patients and median values. C: Correlation between plasma and urinary CD147/basigin levels. Linear approximation line, coefficient of determination $\left(R^{2}\right)$, and $P$ value are shown. D-G: Correlation between proteinuria and plasma (D), and proteinuria and urinary (E) CD147/ basigin values. Correlation between estimated glomerular filtration rate (eGFR) and plasma (F), and eGFR and urinary (G) CD147/basigin levels. Linear approximation lines, coefficients of determination $\left(R^{2}\right)$, and $P$ values are shown. $\mathbf{H}$ : Representative periodic acid-Schiff reagent (PAS) staining in the glomeruli, and immunohistochemical staining of CD147 expression in the glomeruli and tubulointerstitium of patients with FSGS and in a pathological control. Arrows indicate CD147-positive cells. I: Representative images of $\beta_{1}$ integrin and CD147 expression in serial sections from an FSGS patient. Arrows indicate $\beta_{1}$ integrin expression; arrowheads indicate CD147-positive podocytes. $n=95$ (A and B, FSGS); $n=23$ (A and B, pathological controls). ${ }^{* *} P<0.01$. Scale bars: $100 \mu \mathrm{m}$. 
A

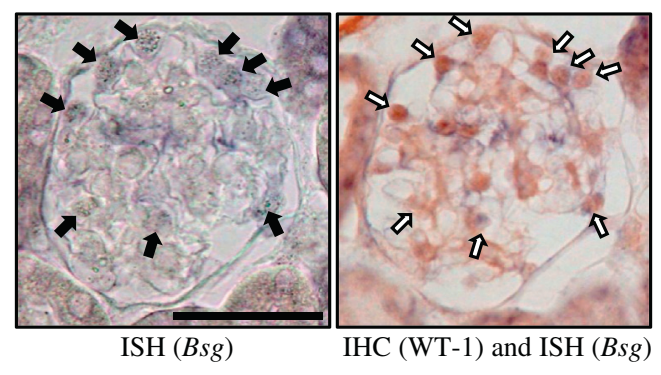

D $\mathrm{Bsg}^{+/+} \quad \mathrm{Bsg}^{/-}$
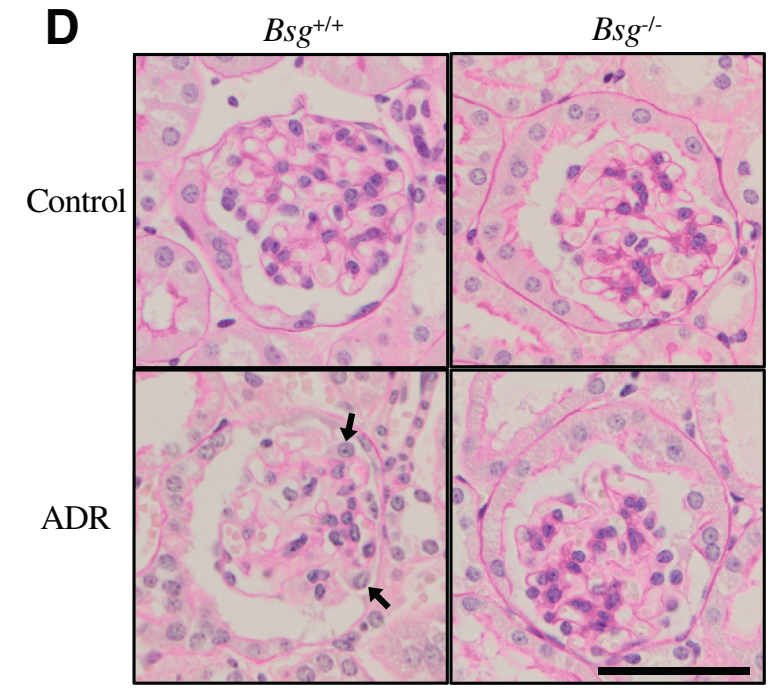

I

Nephrin

$\beta$-actin

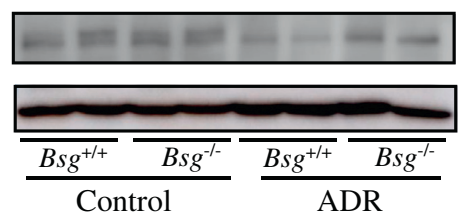

K
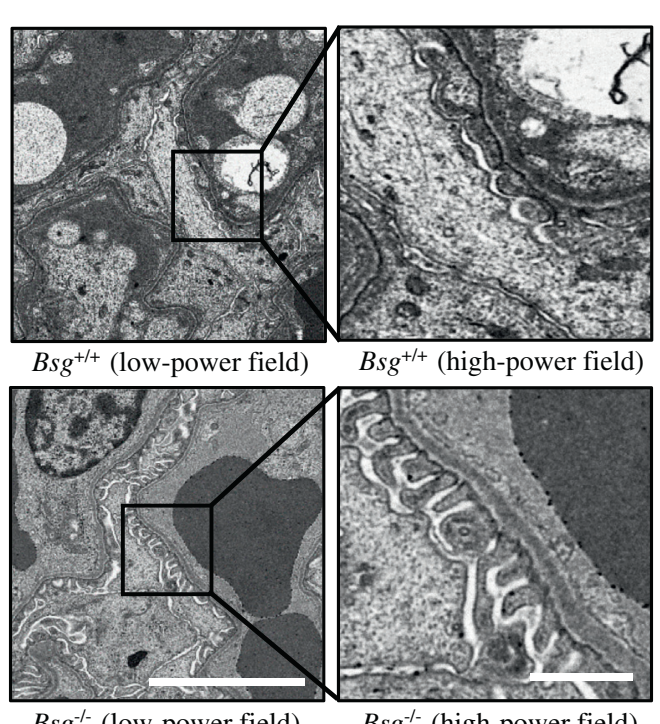

$\mathrm{Bsg}^{-/-}$(low-power field)
B

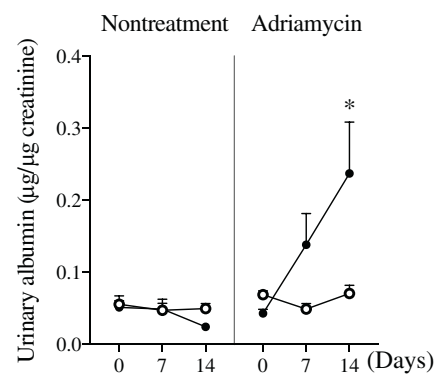

E

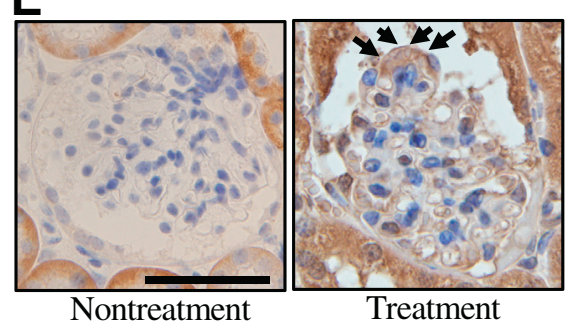

G
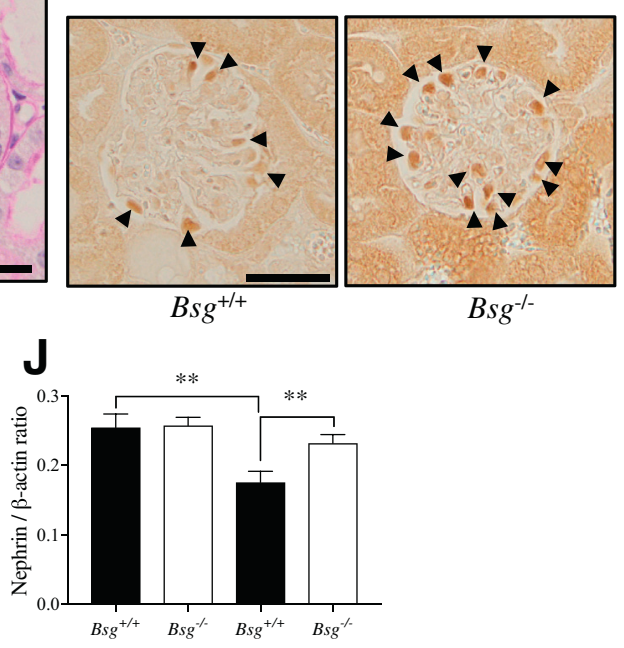

$\mathbf{L}$

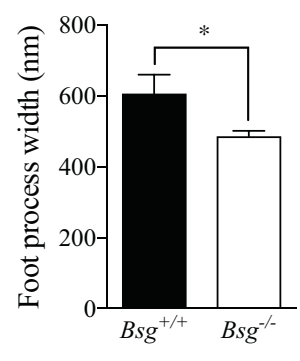

M

Edn1

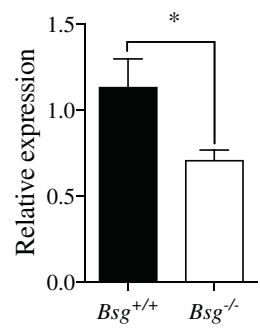

C

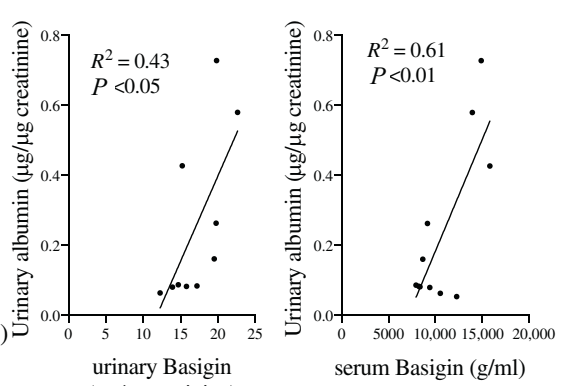

$\mathbf{F}$
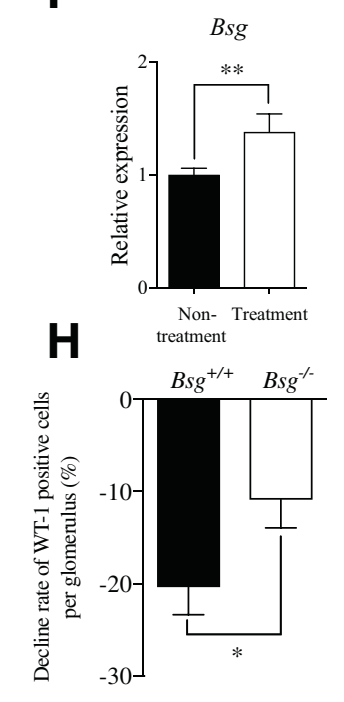
Scientific, Waltham, MA) with siRNA for $B S G$ (GE Healthcare) or negative control siRNA. After 3 days of transfection, the medium was changed to fresh RPMI 1640 medium (Thermo Fisher Scientific) containing $5 \mathrm{ng} / \mathrm{mL}$ recombinant TGF- $\beta$ (R\&D Systems) for $0,12,24,36$, or 48 hours in the time-course study. The total experimental period from the start of transfection to the end of stimulation was 120 hours. Supernatants from cultured podocytes were collected after the different times, and the ET-1 and VEGF levels were measured using an enzyme-linked immunosorbent assay kit according to the manufacturer's instructions (R\&D Systems).

\section{Flow Cytometry Analysis}

Isolated immortalized human podocytes were stained for flow cytometry with the following Abs: mouse anti-human $\beta_{1}$ integrin (BD Biosciences, Piscataway, NJ) or mouse IgG (BD Biosciences), followed by AF488-conjugated goat antimouse IgG1 (Thermo Fisher Scientific). 7AAD (BioLegend, San Diego, CA) was added before flow cytometry to label dead cells. All flow cytometry data were acquired on a BD Canto II (Becton, Dickinson and Company, Franklin Lakes, NJ) and analyzed with FlowJo software version 10 (Tree Star, Ashland, OR).

\section{Migration Assay}

Cell culture inserts with a pore size of $8 \mu \mathrm{m}$ (BD Biosciences, Franklin Lakes, NJ) were placed in the lower compartment, and RPMI 1640 was added. Podocytes $\left(7 \times 10^{3}\right)$ transfected with negative control siRNA or $B S G$ siRNA were placed in 24-well inserts and incubated for 10 hours at $37^{\circ} \mathrm{C}$ to allow migration. Cells that had not migrated were removed from the upper membrane. The number of cells that had migrated were counted in the middle of the membrane.

\section{Wound Healing Assay}

For each experiment, $2.5 \times 10^{4}$ podocytes treated with or without siRNA for $B S G$ were seeded overnight on FN- coated coverslips in 96-well plates. Each well was then scratched with an IncuCyte wound maker (Essen BioScience, Ann Arbor, MI) and washed with phosphate-buffered saline, and then fresh medium was added. At 10 hours after scratching, the numbers of cells that had migrated into the same-sized square fields were counted.

\section{Gelatin Zymography}

Gelatin zymography ${ }^{19}$ was performed with the same amount of conditioned medium from $2.0 \times 10^{4}$ podocytes treated with or without siRNA for $B S G$ on 30-mm plastic dishes to assess MMP-mediated proteolytic activity. Samples of condition medium were loaded onto a $10 \%$ gelatin zymogram protein gel (Thermo Fisher Scientific). Proteins were separated with electrophoresis, and then the gel was treated with $2.5 \%$ Triton X-100 at room temperature for 30 minutes with gentle shaking, followed by incubation at $37^{\circ} \mathrm{C}$ for 24 hours in buffer containing calcium chloride Tris- $\mathrm{HCl}$ for development. The gel was stained with Coomassie Blue, and the density of the bands was quantitated with ImageJ software version 1.48 (NIH).

\section{Statistical Analysis}

Data are the means \pm SEM. For single comparisons, the unpaired $t$-test or the nonparametric $U$-test was used. For multiple comparisons, analysis of variance was used, followed by post hoc least significant difference tests if the initial analysis of variance was significant. Spearman correlation coefficients were calculated to determine the strength of the association between two variables. $P<0.05$ was considered statistically significant.

\section{Results}

\section{Clinicopathological Characteristics of Patients with FSGS}

Proteinuria in 28 patients with FSGS showed a significant correlation with urinary CD147/Bsg levels, but not plasma CD147/Bsg values. ${ }^{23}$ Because the previous study was small, 95 patients with FSGS were enrolled in the present study.

\footnotetext{
Figure 2 Bsg deficiency ameliorates podocyte injury in doxorubicin hydrochloride (ADR)-treated mice. A: Representative images of Bsg mRNA expression in the glomeruli of healthy mice, using in situ hybridization (left panel). Double staining for Bsg mRNA expression with in situ hybridization and WT-1 expression with immunostaining (right panel). Colocalization of Bsg (black arrows) and WT-1 expression (white arrows) in "healthy" glomeruli. B: Time course of albuminuria-to-creatinine ratio in $B s g^{+/+}$and $B s g$-deficient $\left(B s g^{-/-}\right)$mice after ADR treatment. Closed circles, $B s g^{+/+}$mice; open circles: $B s g^{-/-}$mice. C: Correlations between the urinary albumin-creatinine ratio and Bsg levels (left panel), and the urinary albumin/creatinine ratio and serum Bsg levels (right panel) in ADR-treated $\mathrm{Bsg}^{+/+}$mice. D: Representative images of periodic acid-Schiff reagent-stained glomeruli at 14 days in ADR-treated $B s g^{+/+}$and $B s g^{-/-}$ mice. Arrows, podocyte swelling. E: Immunostaining for Bsg protein in the glomeruli of "healthy" or ADR-treated wild-type $\left(B s g^{+/+}\right)$mice. Black arrows, positive areas. F: Glomerular Bsg mRNA expression in ADR-treated mice. G: Immunohistochemistry for WT-1 expression. Black arrowheads, WT-1-positive cells. H: Evaluation of the rate of decline in WT-1-positive cells in the glomeruli of ADR-treated mice. Black bars, $B s g^{+/+}$mice; white bars, Bsg ${ }^{-/-}$mice. I: Nephrin expression in the kidneys at 14 days after ADR treatment as determined by Western blot analysis. J: The intensities of the nephrin bands following normalization to $\beta$-actin. K: Electron micrograph showing the development of foot process effacement in ADR-treated $B s g^{+/+}$mice. L: Quantification of foot process width. M: Endothelin (ET)-1 and transforming growth factor (TGF)- $\beta$ mRNA expression in the glomeruli of ADR-treated mice. Data are expressed as means $\pm \operatorname{SEM}(\mathbf{B}, \mathbf{F}, \mathbf{H}, \mathbf{J}, \mathbf{L}$, and $\mathbf{M}) . n=9$ to $11(\mathbf{C}) ; n=7$ to $11(\mathbf{F}, \mathbf{H}, \mathbf{J}, \mathbf{L}$, and $\mathbf{M}) .{ }^{*} P<0.05$, ${ }^{*} P<0.01$. Scale bars: $50 \mu \mathrm{m}(\mathbf{A}, \mathbf{D}, \mathbf{E}$, and $\mathbf{G}) ; 5.0 \mu \mathrm{m}(\mathbf{K}$, left panels); $0.5 \mu \mathrm{m}$ (K, right panels). IHC, immunohistochemistry; ISH, in situ hybridization.
} 
Table 2 General Characteristics of ADR-Treated Mice

\begin{tabular}{|c|c|c|c|c|}
\hline \multirow[b]{2}{*}{ General characteristics } & \multicolumn{2}{|l|}{ Control mice } & \multicolumn{2}{|l|}{ ADR mice } \\
\hline & $\mathrm{Bsg}^{+/+}$ & $B s g^{-/-}$ & $\mathrm{Bsg}^{+/+}$ & $B s g^{-/-}$ \\
\hline Body weight, day $0, \mathrm{~g}$ & $27.7 \pm 1.0$ & $28.6 \pm 0.6$ & $27.4 \pm 0.5$ & $27.5 \pm 0.6$ \\
\hline Kidney weight/body weight, $\%$ & $0.69 \pm 0.04$ & $0.90 \pm 0.01$ & $0.77 \pm 0.02$ & $0.87 \pm 0.04$ \\
\hline Serum $\mathrm{Cr}, \mathrm{mg} / \mathrm{dL}$ & $0.16 \pm 0.01$ & $0.13 \pm 0.01$ & $0.19 \pm 0.02$ & $0.15 \pm 0.01$ \\
\hline Serum albumin, $\mathrm{mg} / \mathrm{dL}$ & $3.30 \pm 0.12$ & $3.42 \pm 0.06$ & $3.00 \pm 0.16$ & $3.31 \pm 0.13$ \\
\hline
\end{tabular}

Data are expressed as means \pm SEM.

$\mathrm{ADR}$, doxorubicin hydrochloride; $\mathrm{Cr}$, creatinine.

Their characteristics are summarized in Table 1. All participants were Japanese, and slightly more than half $(54.7 \%)$ were male. Both plasma and urinary CD147/Bsg levels were almost twice those of pathologic control patients (Figure 1, A and B). A close relationship between plasma and urinary CD147/Bsg levels was observed (Figure 1C). Of note, both CD147/Bsg values were closely correlated with proteinuria, but the association with the estimated glomerular filtration rate was lower (Figure 1, D-G). These data suggest that plasma and urinary CD147/Bsg levels may not be strongly affected by glomerular filtration function. Immunohistochemical staining of kidney sections from patients with FSGS showed CD147/Bsg protein expression in the glomeruli as well as infiltrating inflammatory cells in the interstitium, but no CD147/Bsg expression in the glomeruli of pathologic controls (Figure 1H). Following staining of serial sections, $\beta_{1}$ integrin and Bsg expression was observed in injured podocytes including adhesions to the Bowman capsule (Figure 1I).

\section{Bsg Deficiency Ameliorates the Development of Proteinuria and Podocyte Injury in ADR-Treated Mice}

Previously, Bsg protein expression was not detected in the glomeruli of kidney sections from nontreated mice. ${ }^{22}$ In the present study, Bsg mRNA expression was observed within the glomeruli as well as the tubular epithelium and vascular endothelium with in situ hybridization (data not shown). Podocyte $B s g$ expression was determined in vivo by co-labeling with $B s g$ and WT-1, a podocyte cell marker. Bsg mRNA expression was colocalized with WT-1 protein expression by immunohistochemistry in normal podocytes (Figure 2A).

To investigate the role of Bsg in the pathogenesis of proteinuric kidney diseases, the degree of glomerular injury was evaluated in $\mathrm{Bsg}^{+/+}$and $\mathrm{Bsg}^{-/-}$mice at 14 days after ADR treatment. ADR-treated $\mathrm{Bsg}^{+/+}$mice tended to show a worse kidney function compared with $B s g^{-1-}$ mice, but the differences were not significant (Table 2). Until 14 days after ADR injection, albuminuria was gradually greater in $\mathrm{Bsg}^{+/+}$mice than in $\mathrm{Bsg}^{-1-}$ mice (Figure 2B). Similar to the profile of patients with FSGS, plasma and urinary Bsg levels showed a significant association with albuminuria (Figure 2C). In addition, $\mathrm{Bsg}^{+/+}$mice with proteinuria appeared to show segmental abnormalities in glomerular architecture and podocyte swelling (Figure 2D). No obvious differences between $\mathrm{Bsg}^{+/+}$and $\mathrm{Bsg}^{-/-}$mice were observed in the tubulointerstitium (data not shown). Healthy podocytes rarely expressed Bsg protein, but they began to express Bsg in the glomerulus upon activation by ADR (Figure 2E). Bs $\mathrm{mRNA}$ expression was also increased in the glomerulus of ADRtreated mice (Figure 2F). The number of macrophages that infiltrated into the glomerulus appeared to be augmented in $\mathrm{Bsg}^{+/+}$mice (data not shown).

Bsg deficiency prevented the decline in the number of WT1 -positive cells and down-regulation of nephrin expression in the kidneys (Figure 2, G-J). In support of these findings, electron microscopy of the glomerular ultrastructure revealed that foot process effacement outside the GBM was present in $\mathrm{Bsg}^{-/-}$mice to a lesser extent than in $\mathrm{Bsg}^{+/+}$mice (Figure 2, $\mathrm{K}$ and $\mathrm{L}$ ). Adequate barrier function in the GBM is maintained by vasoactive factors such as VEGF and ET-1 produced by podocytes. ${ }^{5}$ Glomerular ET-1 (EDNI) mRNA expression increased in $\mathrm{Bsg}^{+/+}$mice, consistent with the profile of proteinuria and podocyte injury (Figure $2 \mathrm{M}$ ). These results suggest that Bsg deficiency suppressed the degree of glomerular injury, which leads to the development of proteinuria in ADRtreated mice.

\section{Albuminuria Is Also Suppressed in L-name-Treated $\mathrm{Bsg}^{-/-}$Mice}

Further experiments were performed to determine whether Bsg deficiency ameliorates podocyte injury and albuminuria in another mouse model representing endothelial dysfunction and hypertension. Bsg depletion in a novel model of glomerular sclerosis induced by L-name, a nitric oxide synthase inhibitor, attenuated the progression of albuminuria (Figure 3A). Although systolic blood pressure in $\mathrm{Bsg}^{+/+}$and $\mathrm{Bsg}^{-/-}$mice increased during the experimental period, no significant differences between the two genotypes were observed until 8 weeks after treatment (Figure 3B). At 12 weeks after L-name treatment, blood pressure in $B s g^{-1-}$ mice was slightly lower than that in $\mathrm{Bsg}^{+/+}$mice. The glomeruli of both $\mathrm{Bsg}^{-/-}$and $\mathrm{Bsg}^{+/+}$mice showed endothelial dysfunction as reported previously. ${ }^{36,37}$ The degrees of podocyte injury and glomerular 
A

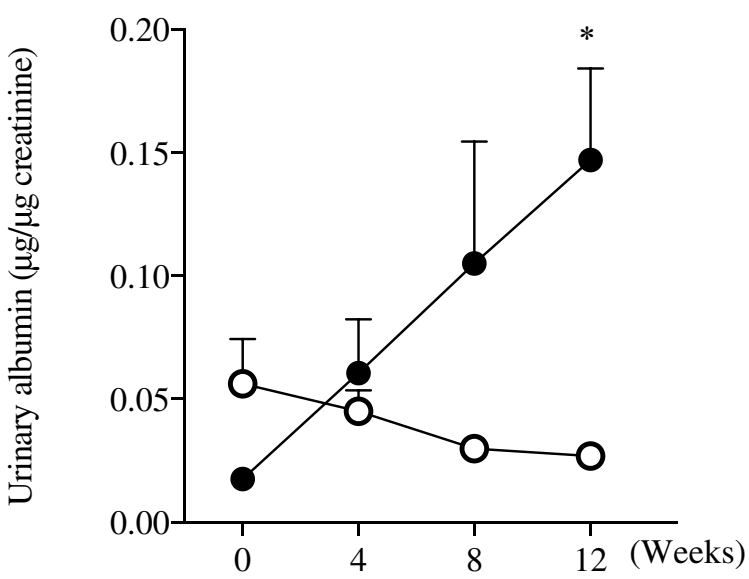

C

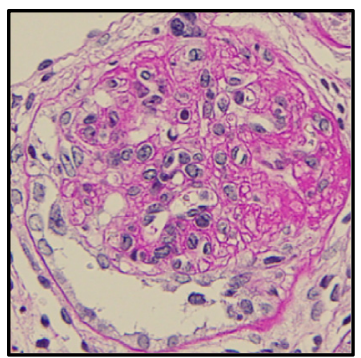

$\mathrm{Bsg}^{+/+}$

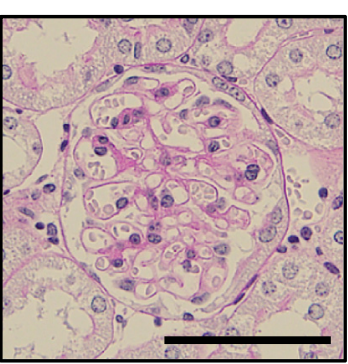

$\mathrm{Bsg}^{-/-}$
B

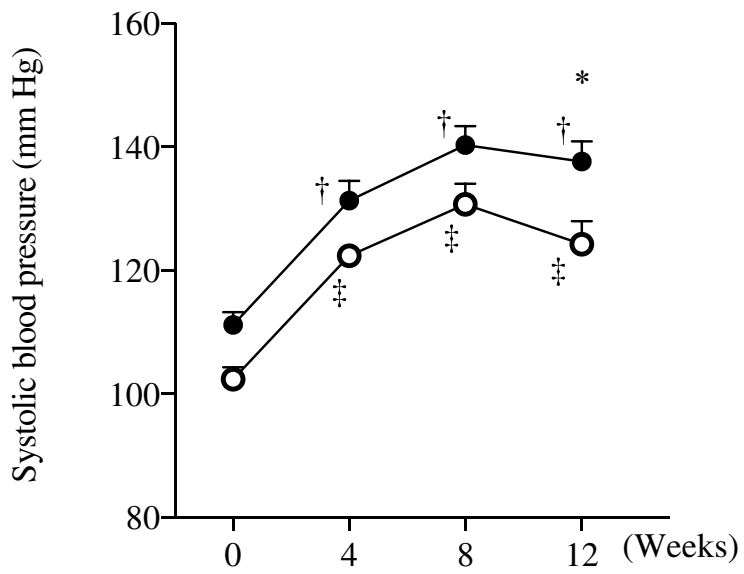

$\mathbf{E}$

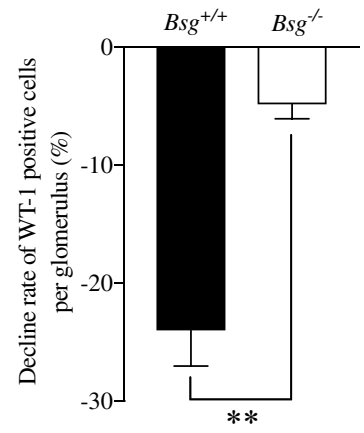

D

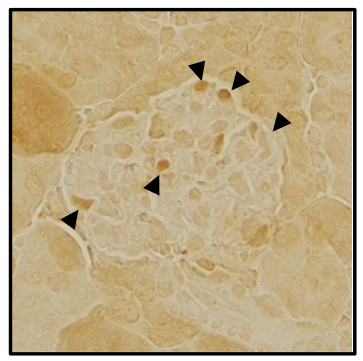

$\mathrm{Bsg}^{+/+}$

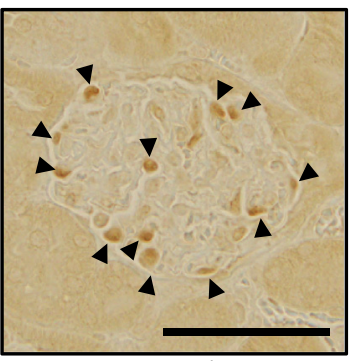

$\mathrm{Bsg}^{-/-}$

$\mathbf{F}$

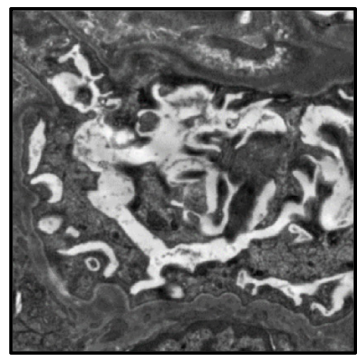

$\mathrm{Bsg}^{+/+}$

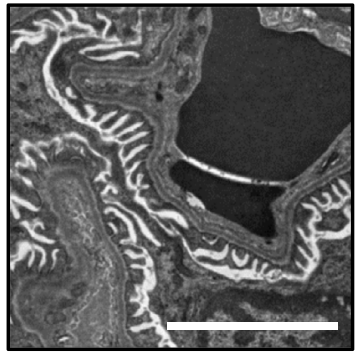

$\mathrm{Bsg}^{-/-}$

Figure 3 L-name-treated $\mathrm{Bsg}^{-/-}$mice show a similar profile as doxorubicin hydrochloride (ADR)-treated $\mathrm{Bsg}^{-/-}$mice. Time course of albuminuria (A) and systolic blood pressure (BP) (B) in L-name-treated $\mathrm{Bsg}^{+/+}$and $\mathrm{Bsg}^{-/-}$mice for 12 weeks after uninephrectomy. Filled circles represent L-name-treated $\mathrm{Bsg}^{+/+}$mice; open circles represent L-name-treated $\mathrm{Bsg}^{-/-}$mice. Representative periodic acid-Schiff reagent (C) and WT-1 (D) staining of $\mathrm{Bsg}^{+/+}$or $\mathrm{Bsg}^{-/-}$ glomeruli treated with L-name at 12 weeks after uninephrectomy. Arrowheads, WT-1-positive cells. E: Evaluation of the rate of decline in WT-1-positive cells based on the podocyte number in the respective control mice. Black bar represents $B s g^{+/+}$mice; white bar represents $B s g^{-/-}$mice. F: Electron micrograph showing foot process effacement in L-name-treated mice. Data are expressed as means $\pm \mathrm{SEM}(\mathbf{A}, \mathbf{B}$, and $\mathbf{E}) . n=11(\mathbf{A}$ and $\mathbf{B}) .{ }^{*} P<0.05$ and ${ }^{* *} P<0.01$ versus $B s g^{-/-}$mice; ${ }^{\dagger} P<0.05$ versus $B s g^{+/+}$BP at pretreatment; ${ }^{\ddagger} P<0.05$ versus $B s g^{-/-}$BP at pretreatment. Scale bars: $5.0 \mu \mathrm{m}$.

sclerosis were suppressed in $\mathrm{Bsg}^{-/-}$mice consistent with the profile of albuminuria (Figure 3, A, C-F).

\section{Podocytes with Silenced Bsg Show Suppression of FAK Activation and Cell Motility}

Podocyte cell spreading and migration are required for the development of proteinuria and focal adhesion in glomerular architecture. ${ }^{2,38}$ Therefore, in cultured mouse podocytes with silenced Bsg, $\beta_{1}$ integrin and phosphorylated FAK expression was evaluated because these proteins play an integral role in cell movement secondary to focal adhesion turnover. ${ }^{39,40}$ The expression of Bsg in cultured podocytes transfected with $B S G$ siRNA was gradually reduced in a time-dependent manner (Figure 4A). 
A

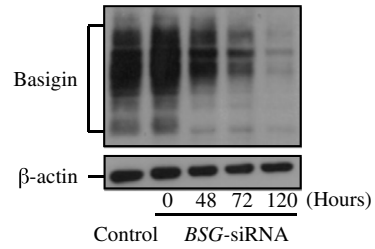

C

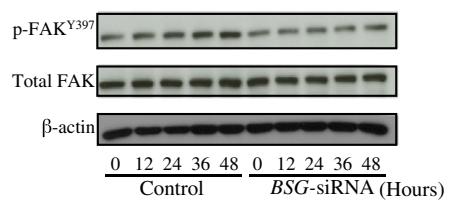

E

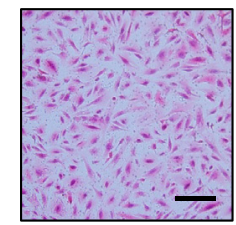

Control

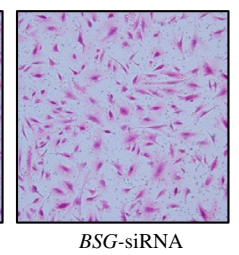

G

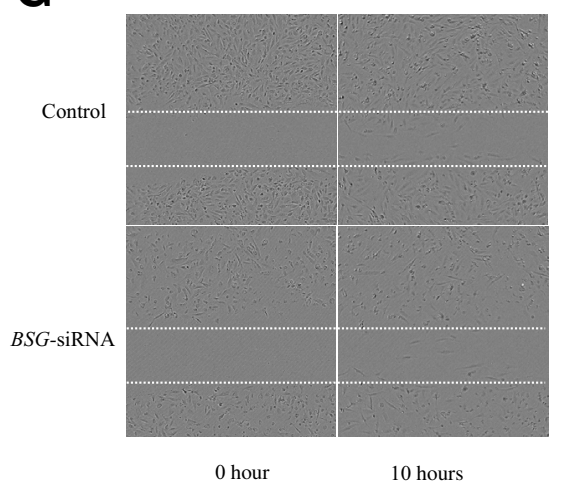

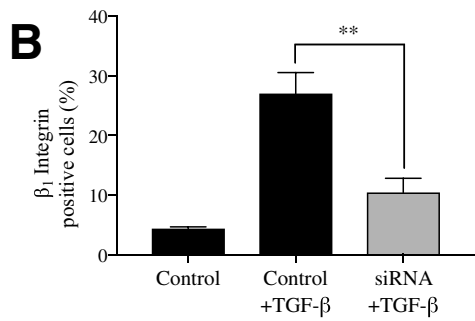

D

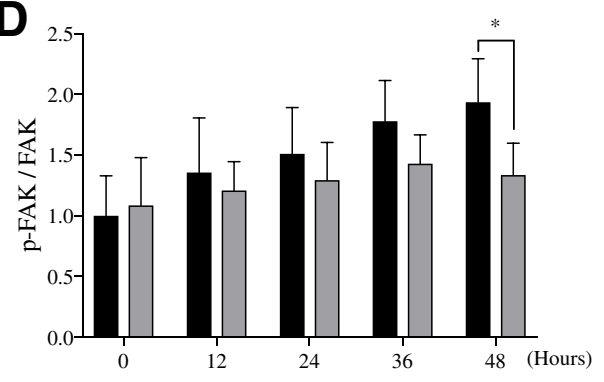

$\mathbf{F}$

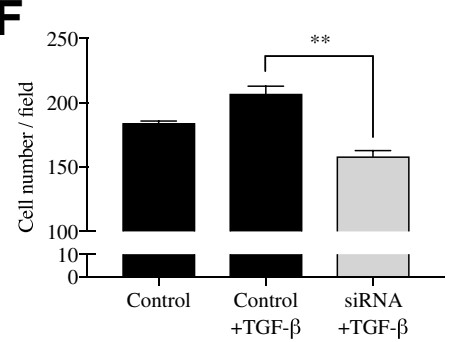

H

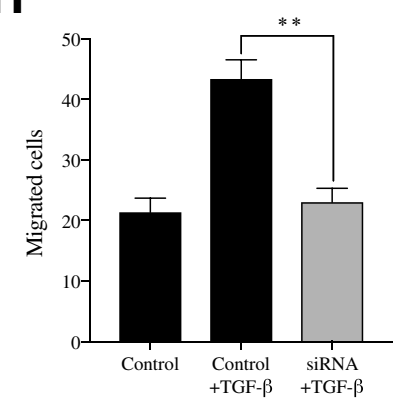

Figure 4 Basigin silencing inhibits directional podocyte movement via focal adhesion kinase phosphorylation ( $p$-FAK). A: Time course of Bsg expression in podocytes transfected with control or BSG siRNA as seen with Western blot analysis. B: The frequency of $\beta_{1}$ integrin $^{+}$podocytes transfected with control or BSG siRNA after $5 \mathrm{ng} /$ $\mathrm{mL}$ TGF- $\beta$ stimulation was determined with flow cytometry. C: Western blot analysis indicates the time course of p-FAK (Y397) in podocyte cells transfected with control or BSG siRNA after $5 \mathrm{ng} /$ $\mathrm{mL}$ TGF- $\beta$ stimulation. D: The intensity of p-FAK bands was normalized to total FAK. Black bars represent control podocytes; gray bars represent BSG siRNA-transfected podocytes. E: Representative images of migrated podocyte cells exposed to TGF- $\beta$ for 10 hours in the migration assay. F: Quantitative analysis of the number of migrated cells. G: Representative images from the wound healing assay. White dashed lines indicate the periphery of the scratch areas. H: Quantification of the results in $\mathbf{G}$. Data are expressed as means $\pm \operatorname{SEM}(\mathbf{B}, \mathbf{D}, \mathbf{F}$, and $\mathbf{H}) . n=3(\mathbf{B}, \mathbf{C}$, and $\mathbf{F}$, independent experiments); $n=5$ (D, independent experiments). ${ }^{*} P<0.05,{ }^{*} P<0.01$. Scale bar, $100 \mu \mathrm{m}$.
TGF- $\beta$ plays a critical role in the pathogenesis of glomerular sclerosis. An in vivo study showed no obvious difference between $\mathrm{Bsg}^{-/-}$and $\mathrm{Bsg}^{+/+}$mice in glomerular TGF- $\beta$ expression (Figure $2 \mathrm{M}$ ). After transfection with $B S G$ siRNA, $\beta_{1}$ integrin expression and FAK phosphorylation were suppressed in $B S G$-silenced podocytes stimulated with $5 \mathrm{ng} / \mathrm{mL}$ TGF- $\beta$ compared with control transfected cells (Figure 4, B-D). To further clarify podocyte migration, podocytes transfected with control siRNA or BSG siRNA were seeded onto the insert filters, and the cells were exposed to $5 \mathrm{ng} / \mathrm{mL}$ TGF- $\beta$. Podocyte migration in response to TGF- $\beta$ was significantly blocked by $B S G$ silencing (Figure 4, E and F). The experiment of podocyte migration was expanded by performing a wound-healing assay, and the number of cells that migrated into the scratched area were calculated. $B S G$ silencing and exposure to TGF- $\beta$ appeared to inhibit directional podocyte movement to fill the wound compared with control podocytes (Figure 4, G and $\mathrm{H}$ ).

The Transition of Cytokines Involved in Intraglomerular Homeostasis

In this setting, a variety of cytokines and chemokines that podocytes produce are involved in the pathogenesis of proteinuria leading to progressive glomerular sclerosis. The expression of VEGF and ET-1, which are implicated in podocyte-to-endothelial cross-communication, was examined in the presence or absence of Bsg. Induction of VEGF 

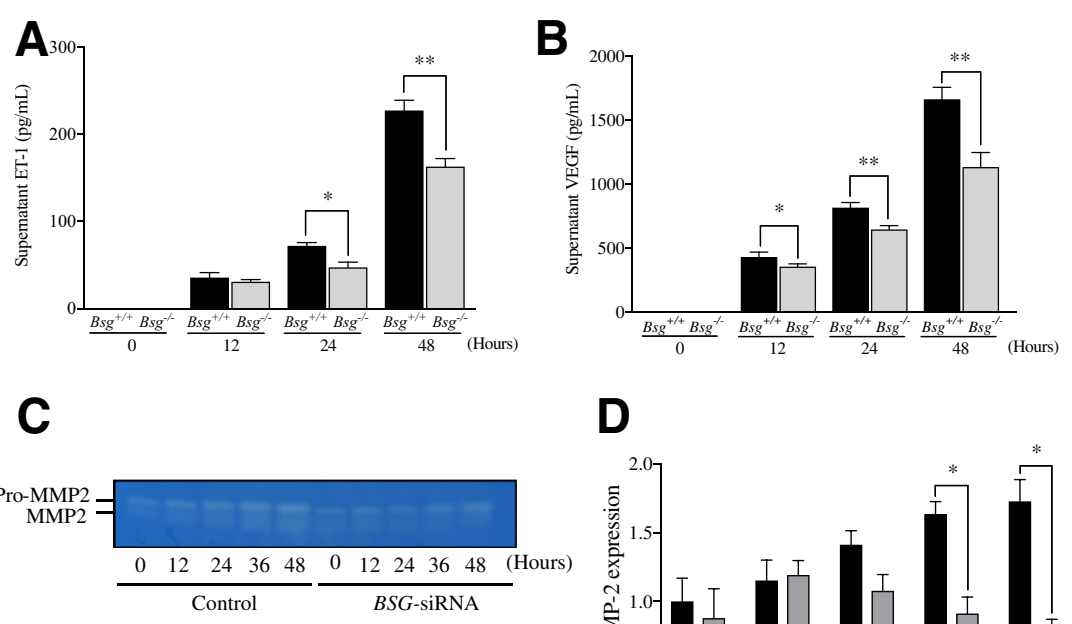

D
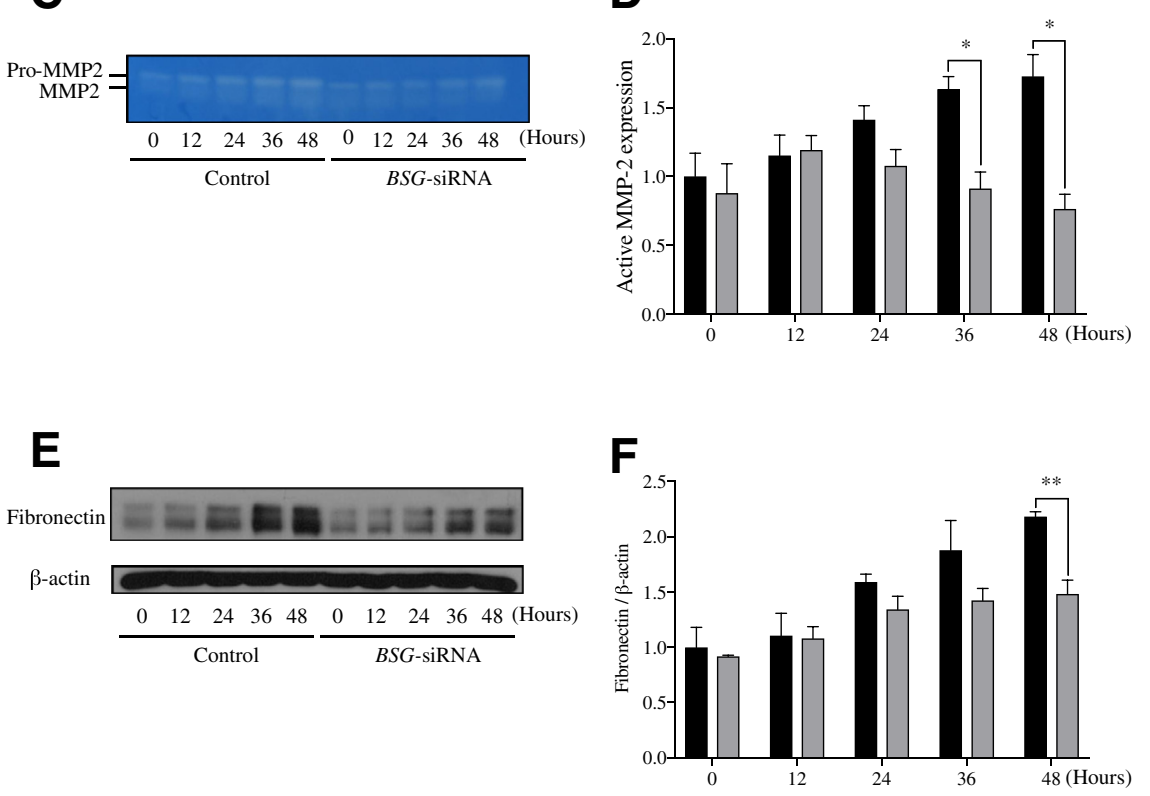

Figure 5 Basigin silencing in podocytes contributes to suppression of several cytokines for disruption of intraglomerular homeostasis following transforming growth factor (TGF) $-\beta$ stimulation. Time course of induction of endothelin (ET)-1 (A) and vascular endothelial growth factor (VEGF)-A (B) in the supernatants of cultured podocyte cells transfected with control or $B S G$ siRNA after exposure to $5 \mathrm{ng} / \mathrm{mL}$ TGF- $\beta$. Black bars, control podocytes; gray bars, BSG siRNAtransfected podocytes. C: Zymographic analysis demonstrates matrix metalloproteinase (MMP) -2 activity in the culture medium of podocytes treated with control and BSG siRNA following TGF$\beta$ stimulation. D: The intensity of active MMP-2 bands. E: Representative Western blot analysis of fibronectin expression in cultured podocytes. F: The intensity of fibronectin bands normalized to $\beta$-actin. Data are expressed as means $\pm \operatorname{SEM}(\mathbf{A}, \mathbf{B}$, $\mathbf{D}$, and $\mathbf{F}) . n=3(\mathbf{F}) ; n=4$ (D, independent experiments); $n=5$ (A and $\mathbf{B}$, independent experiments). ${ }^{*} P<0.05,{ }^{*} P P<0.01$. and ET-1 expression was lower in the supernatants of cultured Bsg-silenced podocytes exposed to TGF- $\beta$ (Figure 5, A and B).

MMP-2 promotes disruption of the GBM and interruption of podocyte integrity. $B s g$ silencing after exposure to TGF- $\beta$ activated MMP-2 to a lesser degree than in control cells that expressed Bsg (Figure 5, C and D). In addition to MMPs, FN is involved in podocyte dedifferentiation and integrinlinked kinase-mediated cross-communication with the GBM. $^{41}$ Similar to the profile of the above cytokines, cellular FN expression was less marked in Bsg-silenced cultured podocytes versus control cells (Figure 5, E and F).

\section{Discussion}

Podocyte injury is caused by maladaptation to various types of stress including mechanical stress, oxidative stress, and biochemical responses, followed by the spread of injury to unique cellular components of glomerular filtration units. ${ }^{38}$ Glomerular endothelial dysfunction also causes podocyte injury by disruption of cross-communication. ${ }^{5}$ According to prior and present clinical studies, healthy podocytes rarely express Bsg protein, but they begin to express Bsg upon activation (Figure 2E). Both urinary and plasma levels of CD147/Bsg showed a closer association with proteinuria in patients with podocytopathy, suggesting that CD147/Bsg induction may be a common feature in human proteinuric kidney diseases. It was therefore assessed how Bsg may be involved in the development of proteinuria. In the present in vivo study using two independent rodent models including ADR-induced nephropathy and L-name-induced endothelial dysfunction, Bsg expressed in injured podocytes was shown to cause podocyte effacement, leading to proteinuria and podocyte injury. Bsg silencing in cultured podocytes suppressed cellular motility and focal adhesion rearrangement via the activation of $\beta_{1}$ integrin-FAK signaling. However, Bsg silencing in glomerular endothelial cells did not affect several molecules related to the maintenance of intraglomerular homeostasis such as VEGF receptor 1 and 2 (data not shown). The collective data supported the idea that CD147/Bsg in podocytes may be a critical molecule in proteinuric kidney diseases.

Activation of $\beta_{1}$ integrin-FAK signaling causes disruption of cell-substrate interactions between podocytes and the GBM because of cellular retraction and movement. ${ }^{39,40}$ Indeed, up-regulation of $\beta_{1}$ integrin expression was found in the early phase of diabetic kidney disease. ${ }^{42}$ A redistribution 


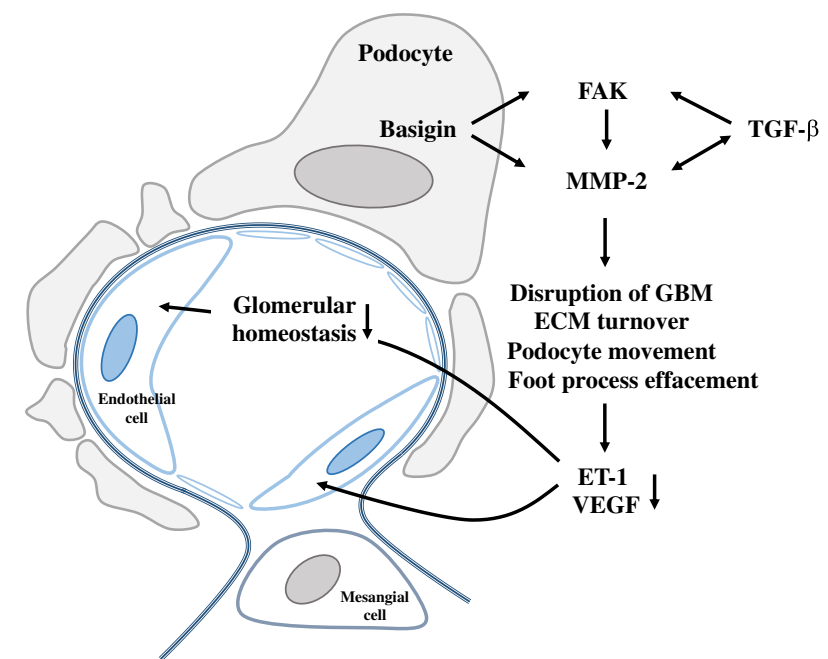

Figure 6 Schematic diagram showing the possible relationship between basigin and podocyte injury, which disrupts maintenance of intraglomerular homeostasis. ECM, extracellular matrix; ET-1, endothelin-1; FAK, focal adhesion kinase; GBM, glomerular basement membrane; MMP, matrix metalloproteinase; TGF- $\beta$, transforming growth factor beta; VEGF, vascular endothelial growth factor.

of $\beta_{1}$ integrin leading to impaired adhesion may be involved in the pathogenesis of podocytopathy. ${ }^{43}$ An elegant study by Ma and colleagues demonstrated that inhibition of podocyte FAK ameliorates the development of proteinuria and foot process effacement. In addition, FAK activation activates MMPs. ${ }^{44,45}$ The in vitro study also demonstrated a beneficial effect of $B s g$ silencing through suppression of $\beta_{1}$ integrin and FAK activation in the presence of TGF- $\beta$ stimulation. Mesenchymal molecules such as MMP-2 and FN in cultured podocytes were reduced by $B s g$ silencing, similar to the profile of phosphorylated FAK, suggesting that Bsg may participate in a phenotypic conversion during podocyte injury that is characterized by defects in epithelial features. During the process of carcinoma metastasis, Bsg interacts with $\beta_{1}$ integrin and then activates downstream FAK/PI3K signaling. ${ }^{27}$ In this setting, interestingly, depolarization of the epithelium appears to be caused by Bsg. ${ }^{46}$ The function of Bsg in hepatocellular carcinoma progress may be partially related to the spread of podocyte injury. Some investigators have documented that podocytes undergo a phenotypic transition after injury. ${ }^{41,47}$ Considering that MMPs activate TGF- $\beta$ and that Bsg is a strong inducer of MMPs, ${ }^{19,48}$ a molecular circuit may be present for podocyte injury that includes signaling molecules such as Bsg, TGF- $\beta, \beta_{1}$ integrin, FAK, MMPs, and FN. The molecular details by which Bsg induces mesenchymal transition in podocytes require further elucidation. In support of these data and consistent with previous reports, Bsg plays a deleterious role in podocyte injury by activating FAK-MMP signaling in proteinuric kidney diseases.

Growing evidence supports the idea that signaling molecules mediate the maintenance of intraglomerular function in an autocrine or paracrine manner. Inappropriate regulation of VEGF and ET-1 derived from podocytes can lead to endothelial dysfunction, including endothelial cell swelling and loss of the glycocalyx. ${ }^{5,6,38}$ Indeed, ET-1 release from podocytes causes mitochondrial dysfunction in adjacent endothelial cells and eventually results in the exacerbation of podocyte apoptosis and albuminuria. ${ }^{49}$ In the present study, both expression of VEGF and ET-1 was higher in cultured podocytes without $B s g$ silenced that were treated with TGF- $\beta$. Glomerular endothelial injury precedes podocyte apoptosis in endothelial nitric oxide synthase-deficient mice, ${ }^{50}$ which show similar characteristics to L-name-treated mice in this experiment. Podocytes may be susceptible to injury by various stimuli and types of stress, regardless of the primary or secondary glomerular diseases. Thus, targeting the crosscommunication between podocytes and the endothelium as well as the various signaling molecules may represent new therapeutic opportunities.

Clinical studies of a wide range of kidney diseases with proteinuria, including lupus nephritis, acute kidney injury, $\operatorname{IgA}$ nephropathy, FSGS, and diabetic kidney disease, have demonstrated the induction of CD147/Bsg protein in glomerular components in proportion to the progress of disease activity. ${ }^{21-23}$ Here, it was demonstrated that Bsg production in the early phase of podocytopathy causes podocyte effacement, which promotes proteinuria. In this setting, Bsg may be involved in a physiological positive feedback loop that accelerates podocyte cell motility and depolarization (Figure 6).

This study has a few limitations. Podocyte-specific deletion of $B s g$ in mice was not performed, and primary cultured podocytes were not used. Because one phenotype of $B s g$-deficient mice is male and female infertility, creating and maintaining a conditional knockout mouse strain is difficult. Because the mice used are a mixed strain that includes $129 / \mathrm{SV}$, susceptibility to ADR may be higher compared with other mouse strains. In a preliminary study, higher dosages of ADR resulted in very low survival rates accompanied by marked glomerular injuries such as severe global glomerulosclerosis (data not shown). To investigate a critical role for Bsg in the early phase of proteinuric kidney diseases, the dosage administered to these mice was therefore set at $16 \mathrm{mg} / \mathrm{kg}$ body weight in the present study.

\section{Conclusions}

In conclusion, the current study suggests that Bsg silencing may be an attractive therapeutic strategy for the maintenance of podocytes via suppression of $\beta_{1}$ integrin-FAK-MMP signaling. These results may open new avenues in the treatment of patients with proteinuric kidney diseases.

\section{Acknowledgments}

We thank Noriyuki Suzuki, Naoko Asano, and Yuriko Sawa for their excellent technical assistance and Hitomi Aoyama for secretarial assistance. 


\section{References}

1. Sarnak MJ, Levey AS, Schoolwerth AC, Coresh J, Culleton B, Hamm LL, McCullough PA, Kasiske BL, Kelepouris E, Klag MJ, Parfrey P, Pfeffer M, Raij L, Spinosa DJ, Wilson PW; American Heart Association Councils on Kidney in Cardiovascular Disease, High Blood Pressure Research, Clinical Cardiology, and Epidemiology and Prevention: Kidney disease as a risk factor for development of cardiovascular disease: a statement from the American Heart Association Councils on Kidney in Cardiovascular Disease, High Blood Pressure Research, Clinical Cardiology, and Epidemiology and Prevention. Circulation 2003, 108:2154-2169

2. Shankland SJ: The podocyte's response to injury: role in proteinuria and glomerulosclerosis. Kidney Int 2006, 69:2131-2147

3. Bansal N, Zelnick LR, Alonso A, Benjamin EJ, de Boer IH, Deo R, Katz R, Kestenbaum B, Mathew J, Robinson-Cohen C, Sarnak MJ, Shlipak MG, Sotoodehnia N, Young B, Heckbert SR: eGFR and albuminuria in relation to risk of incident atrial fibrillation: a metaanalysis of the Jackson Heart Study, the Multi-Ethnic Study of Atherosclerosis, and the Cardiovascular Health Study. Clin J Am Soc Nephrol 2017, 12:1386-1398

4. Hayashi K, Takayama M, Kanda T, Kashiwagi K, Hishikawa A, Iwao Y, Itoh H: Association of kidney dysfunction with asymptomatic cerebrovascular abnormalities in a Japanese population with health checkups. Circ J 2017, 81:1191-1197

5. Dimke H, Maezawa Y, Quaggin SE: Crosstalk in glomerular injury and repair. Curr Opin Nephrol Hypertens 2015, 24:231-238

6. Eremina V, Jefferson JA, Kowalewska J, Hochster H, Haas M, Weisstuch J, Richardson C, Kopp JB, Kabir MG, Backx PH, Gerber HP, Ferrara N, Barisoni L, Alpers CE, Quaggin SE: VEGF inhibition and renal thrombotic microangiopathy. N Engl J Med 2008, 358:1129-1136

7. Sekiuchi M, Kudo A, Nakabayashi K, Kanai-Azuma M, Akimoto Y, Kawakami H, Yamada A: Expression of matrix metalloproteinases 2 and 9 and tissue inhibitors of matrix metalloproteinases 2 and 1 in the glomeruli of human glomerular diseases: the results of studies using immunofluorescence, in situ hybridization, and immunoelectron microscopy. Clin Exp Nephrol 2012, 16:863-874

8. Li SY, Huang PH, Yang AH, Tarng DC, Yang WC, Lin CC, Chen JW, Schmid-Schonbein G, Lin SJ: Matrix metalloproteinase-9 deficiency attenuates diabetic nephropathy by modulation of podocyte functions and dedifferentiation. Kidney Int 2014, 86:358-369

9. Kosugi T, Maeda K, Sato W, Maruyama S, Kadomatsu K: CD147 (EMMPRIN/Basigin) in kidney diseases: from an inflammation and immune system viewpoint. Nephrol Dial Transplant 2015, 30: $1097-1103$

10. Muramatsu T, Miyauchi T: Basigin (CD147): a multifunctional transmembrane protein involved in reproduction, neural function, inflammation and tumor invasion. Histol Histopathol 2003, 18: 981-987

11. Nabeshima K, Iwasaki H, Koga K, Hojo H, Suzumiya J, Kikuchi M: Emmprin (basigin/CD147): matrix metalloproteinase modulator and multifunctional cell recognition molecule that plays a critical role in cancer progression. Pathol Int 2006, 56:359-367

12. Yurchenko V, Constant S, Bukrinsky M: Dealing with the family: CD147 interactions with cyclophilins. Immunology 2006, 117: 301-309

13. Ruiz S, Castro-Castro A, Bustelo XR: CD147 inhibits the nuclear factor of activated T-cells by impairing Vav1 and Rac1 downstream signaling. J Biol Chem 2008, 283:5554-5566

14. Crosnier C, Bustamante LY, Bartholdson SJ, Bei AK, Theron M, Uchikawa M, Mboup S, Ndir O, Kwiatkowski DP, Duraisingh MT, Rayner JC, Wright GJ: Basigin is a receptor essential for erythrocyte invasion by Plasmodium falciparum. Nature 2011, 480:534-537

15. Solstad T, Bains SJ, Landskron J, Aandahl EM, Thiede B, Tasken K, Torgersen KM: CD147 (Basigin/Emmprin) identifies
FoxP3 + CD45RO + CTLA4+-activated human regulatory $\mathrm{T}$ cells. Blood 2011, 118:5141-5151

16. Chen S, Kadomatsu K, Kondo M, Toyama Y, Toshimori K, Ueno S, Miyake Y, Muramatsu T: Effects of flanking genes on the phenotypes of mice deficient in basigin/CD147. Biochem Biophys Res Commun 2004, 324:147-153

17. Igakura T, Kadomatsu K, Kaname T, Muramatsu H, Fan QW, Miyauchi T, Toyama Y, Kuno N, Yuasa S, Takahashi M, Senda T, Taguchi O, Yamamura K, Arimura K, Muramatsu T: A null mutation in basigin, an immunoglobulin superfamily member, indicates its important roles in peri-implantation development and spermatogenesis. Dev Biol 1998, 194:152-165

18. Kato N, Yuzawa Y, Kosugi T, Hobo A, Sato W, Miwa Y, Sakamoto K, Matsuo S, Kadomatsu K: The E-selectin ligand basi$\mathrm{gin} / \mathrm{CD} 147$ is responsible for neutrophil recruitment in renal ischemia/reperfusion. J Am Soc Nephrol 2009, 20:1565-1576

19. Kato N, Kosugi T, Sato W, Ishimoto T, Kojima H, Sato Y, Sakamoto K, Maruyama S, Yuzawa Y, Matsuo S, Kadomatsu K: Basigin/CD147 promotes renal fibrosis after unilateral ureteral obstruction. Am J Pathol 2011, 178:572-579

20. Maeda K, Kosugi T, Sato W, Kojima H, Sato Y, Kamimura D, Kato N, Tsuboi N, Yuzawa Y, Matsuo S, Murakami M, Maruyama S, Kadomatsu K: CD147/basigin limits lupus nephritis and Th17 cell differentiation in mice by inhibiting the interleukin-6/STAT-3 pathway. Arthritis Rheumatol 2015, 67:2185-2195

21. Nagaya H, Kosugi T, Maeda-Hori M, Maeda K, Sato Y, Kojima H, Hayashi H, Kato N, Ishimoto T, Sato W, Yuzawa Y, Matsuo S, Kadomatsu K, Maruyama S: CD147/basigin reflects renal dysfunction in patients with acute kidney injury. Clin Exp Nephrol 2014, 18: 746-754

22. Maeda-Hori M, Kosugi T, Kojima H, Sato W, Inaba S, Maeda K, Nagaya $\mathrm{H}$, Sato $\mathrm{Y}$, Ishimoto $\mathrm{T}$, Ozaki T, Tsuboi N, Muro $\mathrm{Y}$, Yuzawa Y, Imai E, Johnson RJ, Matsuo S, Kadomatsu K, Maruyama S: Plasma CD147 reflects histological features in patients with lupus nephritis. Lupus 2014, 23:342-352

23. Mori Y, Masuda T, Kosugi T, Yoshioka T, Hori M, Nagaya H, Maeda K, Sato Y, Kojima H, Kato N, Ishimoto T, Katsuno T, Yuzawa Y, Kadomatsu K, Maruyama S: The clinical relevance of plasma CD147/basigin in biopsy-proven kidney diseases. Clin Exp Nephrol 2018, 22:815-824

24. Sun J, Hemler ME: Regulation of MMP-1 and MMP-2 production through CD147/extracellular matrix metalloproteinase inducer interactions. Cancer Res 2001, 61:2276-2281

25. Yurchenko V, Constant S, Eisenmesser E, Bukrinsky M: CyclophilinCD147 interactions: a new target for anti-inflammatory therapeutics. Clin Exp Immunol 2010, 160:305-317

26. Wang CH, Yao H, Chen LN, Jia JF, Wang L, Dai JY, Zheng ZH, Chen ZN, Zhu P: CD147 induces angiogenesis through a vascular endothelial growth factor and hypoxia-inducible transcription factor 1alpha-mediated pathway in rheumatoid arthritis. Arthritis Rheum 2012, 64:1818-1827

27. Wu J, Li Y, Dang YZ, Gao HX, Jiang JL, Chen ZN: HAb18G/CD147 promotes radioresistance in hepatocellular carcinoma cells: a potential role for integrin beta1 signaling. Mol Cancer Ther 2015, 14:553-563

28. Maeda K, Otomo K, Yoshida N, Abu-Asab MS, Ichinose K, Nishino T, Kono M, Ferretti A, Bhargava R, Maruyama S, Bickerton S, Fahmy TM, Tsokos MG, Tsokos GC: CaMK4 compromises podocyte function in autoimmune and nonautoimmune kidney disease. J Clin Invest 2018, 128:3445-3459

29. Suda O, Tsutsui M, Morishita T, Tanimoto A, Horiuchi M, Tasaki H, Huang PL, Sasaguri Y, Yanagihara N, Nakashima Y: Long-term treatment with $\mathrm{N}$ (omega)-nitro-L-arginine methyl ester causes arteriosclerotic coronary lesions in endothelial nitric oxide synthasedeficient mice. Circulation 2002, 106:1729-1735

30. Kosugi T, Heinig M, Nakayama T, Matsuo S, Nakagawa T: eNOS knockout mice with advanced diabetic nephropathy have less benefit 
from renin-angiotensin blockade than from aldosterone receptor antagonists. Am J Pathol 2010, 176:619-629

31. Sato Y, Sato W, Maruyama S, Wilcox CS, Falck JR, Masuda T, Kosugi T, Kojima H, Maeda K, Furuhashi K, Ando M, Imai E, Matsuo S, Kadomatsu K: Midkine regulates BP through cytochrome P450-derived eicosanoids. J Am Soc Nephrol 2015, 26:1806-1815

32. Koop K, Eikmans M, Baelde HJ, Kawachi H, De Heer E, Paul LC, Bruijn JA: Expression of podocyte-associated molecules in acquired human kidney diseases. J Am Soc Nephrol 2003, 14:2063-2071

33. Kojima H, Kosugi T, Sato W, Sato Y, Maeda K, Kato N, Kato K, Inaba S, Ishimoto T, Tsuboi N, Matsuo S, Maruyama S, Yuzawa Y, Kadomatsu K: Deficiency of growth factor midkine exacerbates necrotizing glomerular injuries in progressive glomerulonephritis. Am J Pathol 2013, 182:410-419

34. Masuda T, Maeda K, Sato W, Kosugi T, Sato Y, Kojima H, Kato N, Ishimoto $\mathrm{T}$, Tsuboi $\mathrm{N}$, Uchimura $\mathrm{K}$, Yuzawa $\mathrm{Y}$, Maruyama $\mathrm{S}$, Kadomatsu K: Growth factor midkine promotes T-cell activation through nuclear factor of activated $\mathrm{T}$ cells signaling and Th1 cell differentiation in lupus nephritis. Am J Pathol 2017, 187:740-751

35. Ishimoto T, Shimada M, Gabriela G, Kosugi T, Sato W, Lee PY, Lanaspa MA, Rivard C, Maruyama S, Garin EH, Johnson RJ: Tolllike receptor 3 ligand, polyIC, induces proteinuria and glomerular CD80, and increases urinary CD80 in mice. Nephrol Dial Transplant 2013, 28:1439-1446

36. Kosugi T, Heinig M, Nakayama T, Connor T, Yuzawa Y, Li Q, Hauswirth WW, Grant MB, Croker BP, Campbell-Thompson M, Zhang L, Atkinson MA, Segal MS, Nakagawa T: Lowering blood pressure blocks mesangiolysis and mesangial nodules, but not tubulointerstitial injury, in diabetic eNOS knockout mice. Am J Pathol 2009, 174:1221-1229

37. Nakagawa T, Tanabe K, Croker BP, Johnson RJ, Grant MB, Kosugi T, Li Q: Endothelial dysfunction as a potential contributor in diabetic nephropathy. Nat Rev Nephrol 2011, 7:36-44

38. Nagata M: Podocyte injury and its consequences. Kidney Int 2016, $89: 1221-1230$

39. Mitra SK, Hanson DA, Schlaepfer DD: Focal adhesion kinase: in command and control of cell motility. Nat Rev Mol Cell Biol 2005, 6; $56-68$

40. Ma H, Togawa A, Soda K, Zhang J, Lee S, Ma M, Yu Z, Ardito T, Czyzyk J, Diggs L, Joly D, Hatakeyama S, Kawahara E, Holzman L, Guan JL, Ishibe S: Inhibition of podocyte FAK protects against proteinuria and foot process effacement. J Am Soc Nephrol 2010, 21: $1145-1156$

41. Kang YS, Li Y, Dai C, Kiss LP, Wu C, Liu Y: Inhibition of integrinlinked kinase blocks podocyte epithelial-mesenchymal transition and ameliorates proteinuria. Kidney Int 2010, 78:363-373

42. Sawada K, Toyoda M, Kaneyama N, Shiraiwa S, Moriya H, Miyatake H, Tanaka E, Yamamoto N, Miyauchi M, Kimura M, Wada T, Fukagawa M: Upregulation of alpha3beta1-integrin in podocytes in early-stage diabetic nephropathy. J Diabetes Res 2016, 2016:9265074

43. Robins R, Baldwin C, Aoudjit L, Cote JF, Gupta IR, Takano T: Rac1 activation in podocytes induces the spectrum of nephrotic syndrome. Kidney Int 2017, 92:349-364

44. Hsia DA, Mitra SK, Hauck CR, Streblow DN, Nelson JA, Ilic D, Huang S, Li E, Nemerow GR, Leng J, Spencer KS, Cheresh DA, Schlaepfer DD: Differential regulation of cell motility and invasion by FAK. J Cell Biol 2003, 160:753-767

45. Visse R, Nagase H: Matrix metalloproteinases and tissue inhibitors of metalloproteinases: structure, function, and biochemistry. Circ Res 2003, 92:827-839

46. Lu M, Wu J, Hao ZW, Shang YK, Xu J, Nan G, Li X, Chen ZN, Bian H: Basolateral CD147 induces hepatocyte polarity loss by Ecadherin ubiquitination and degradation in hepatocellular carcinoma progress. Hepatology 2018, 68:317-332

47. Li Y, Kang YS, Dai C, Kiss LP, Wen X, Liu Y: Epithelial-tomesenchymal transition is a potential pathway leading to podocyte dysfunction and proteinuria. Am J Pathol 2008, 172:299-308

48. Karsdal MA, Larsen L, Engsig MT, Lou H, Ferreras M, Lochter A, Delaisse JM, Foged NT: Matrix metalloproteinase-dependent activation of latent transforming growth factor-beta controls the conversion of osteoblasts into osteocytes by blocking osteoblast apoptosis. J Biol Chem 2002, 277:44061-44067

49. Daehn I, Casalena G, Zhang T, Shi S, Fenninger F, Barasch N, Yu L, D’Agati V, Schlondorff D, Kriz W, Haraldsson B, Bottinger EP: Endothelial mitochondrial oxidative stress determines podocyte depletion in segmental glomerulosclerosis. J Clin Invest 2014, 124: $1608-1621$

50. Sun YB, Qu X, Zhang X, Caruana G, Bertram JF, Li J: Glomerular endothelial cell injury and damage precedes that of podocytes in adriamycin-induced nephropathy. PLoS One 2013, 8: e55027 